The Astrophysical Journal, 661: L123-L126, 2007 June 1

(C) 2007. The American Astronomical Society. All rights reserved. Printed in U.S.A.

\title{
THE PECULIAR VELOCITIES OF LOCAL TYPE Ia SUPERNOVAE AND THEIR IMPACT ON COSMOLOGY
}

\author{
JAMES D. NeILl \\ California Institute of Technology, 1200 East California Boulevard, Pasadena, CA 91125; neill@ srl.caltech.edu \\ Michael J. Hudson \\ University of Waterloo, 200 University Avenue West, Waterloo, ON N2L 3G1, Canada; mjhudson@uwaterloo.ca \\ AND \\ Alex Conley \\ University of Toronto, 60 Saint George Street, Toronto ON M5S 3H8, Canada; conley@astro.utoronto.ca \\ Received 2007 March 5; accepted 2007 April 12; published 2007 May 11
}

\begin{abstract}
We quantify the effect of Type Ia supernova (SN Ia) peculiar velocities on the derivation of cosmological parameters. The published distant and local SNe Ia used for the Supernova Legacy Survey first-year cosmology report form the sample for this study. While previous work has assumed that the local SNe are at rest in the CMB frame (the no-flow assumption), we test this assumption by applying peculiar velocity corrections to the local SNe using three different flow models. The models are based on the IRAS PSCz galaxy redshift survey, have varying $\beta=\Omega_{m}^{0.6} / b$, and reproduce the Local Group motion in the CMB frame. These data sets are then fit for $w, \Omega_{m}$, and $\Omega_{\Lambda}$ using flatness or $\Lambda \mathrm{CDM}$ and a BAO prior, and the $\chi^{2}$ statistic is used to examine the effect of the velocity corrections on the quality of the fits. The most favored model is the $\beta=0.5$ model, which produces a fit significantly better than the no-flow assumption, consistent with previous peculiar velocity studies. By comparing the no-flow assumption with the favored models, we derive the largest potential systematic error in $w$ caused by ignoring peculiar velocities, $\Delta w=+0.04$. For $\Omega_{\Lambda}$ the potential error is $\Delta \Omega_{\Lambda}=-0.04$, and for $\Omega_{m}$ the potential error is $\Delta \Omega_{m}<+0.01$. The favored flow model $(\beta=0.5)$ produces the following cosmological parameters: $w=-1.08_{-0.08}^{+0.09}, \Omega_{m}=0.27_{-0.02}^{+0.02}$ assuming a flat cosmology, and $\Omega_{\Lambda}=0.80_{-0.07}^{+0.08}$ and $\Omega_{m}=0.27_{-0.02}^{+0.02}$ for a $w=-1(\Lambda \mathrm{CDM})$ cosmology.
\end{abstract}

Subject headings: galaxies: distances and redshifts - large-scale structure of universe — supernovae: general

\section{INTRODUCTION}

Dark energy has challenged our knowledge of fundamental physics since the direct evidence for its existence was discovered using Type Ia supernovae (SNe Ia; Riess et al. 1998; Perlmutter et al. 1999). Because there are currently no compelling theoretical explanations for dark energy, the correct emphasis, as pointed out by the Dark Energy Task Force (DETF; Albrecht et al. 2006), is on refining our observations of the accelerated expansion of the universe. Recommendation $\mathrm{V}$ from the DETF report (Albrecht et al. 2006) calls for an exploration of the systematic effects that could impair the needed observational refinements.

A couple of recent studies (Hui \& Greene 2006; Cooray \& Caldwell 2006) point out that the redshift lever arm needed to accurately measure the universal expansion requires the use of a local sample but that coherent large-scale local $(z<0.2)$ peculiar velocities add additional uncertainty to the Hubble diagram and hence to the derived cosmological parameters.

Current analyses (e.g., Astier et al. 2006; Riess et al. 2007; Wood-Vasey et al. 2007) of the cosmological parameters do not attempt to correct for the effect of local peculiar velocities. As briefly noted by Hui \& Greene (2006) and Cooray \& Caldwell (2006), it is possible to use local data to measure the local velocity field and hence limit the impact on the derived cosmological parameters. Measurements of the local velocity field have improved to the point where there is consistency among surveys and methods (Hudson 2003; Hudson et al. 2004; Radburn-Smith et al. 2004; Pike \& Hudson 2005; Sarkar et al. 2006). Type Ia supernova peculiar velocities have been studied recently by Radburn-Smith et al. (2004), Jha et al. (2007), Haugboelle et al. (2006), Watkins \& Feldman (2007), and others. Their results demonstrate that the local flows derived from $\mathrm{SNe}$ are in agree- ment with those derived from other distance indicators, such as the Tully-Fisher relation and the fundamental plane. Our aim is to use the current knowledge of the local peculiar motions to correct local SNe and, together with a homogeneous set of distant $\mathrm{SNe}$, fit for cosmological parameters and measure the effect of the corrections on the cosmological fits.

To produce this measurement, we analyze the local and distant SN Ia sample used in the first-year cosmology results from the Supernova Legacy Survey (SNLS; Astier et al. 2006, hereafter A06). This sample is composed of 44 local SNe (A06, Table 8; Hamuy et al. 1996; Riess et al. 1999; Krisciunas et al. 2001; Jha 2002; Strolger et al. 2002; Altavilla et al. 2004; Krisciunas et al. 2004a, 2004b) and 71 distant SNe (A06, Table 9). The distant $\mathrm{SNe}$ are the largest homogeneous set currently in the literature. The local sample spans the redshift range $0.015<z<0.125$ and was selected to have good light-curve sampling (A06, § 5.2). Using three different models encompassing the range of plausible local large-scale flow, we assign and correct for the peculiar velocity of each local SN. We then refit the entire sample for $w$, $\Omega_{m}$, and $\Omega_{\Lambda}$ to assess the systematics due to the peculiar velocity field and to asses the change in the quality of the resulting fits.

\section{PECULIAR VELOCITY MODELS}

Peculiar velocities, $\boldsymbol{v}$, arise due to inhomogeneities in the mass density and hence in the expansion. Their effect is to perturb the observed redshifts from their cosmological values: $c z_{\mathrm{CMB}}=c z+\boldsymbol{v} \cdot \hat{\boldsymbol{r}}$, where $c z$ is the cosmological redshift that the $\mathrm{SN}$ would have in the absence of peculiar velocities. With the advent of all-sky galaxy redshift surveys, it is possible to predict peculiar velocities from the galaxy distribution provided one knows $\beta=f(\Omega) / b$, where $b$ is a linear biasing parameter relating fluctuations in the galaxy density, $\delta$, to fluctuations in 
TABLE 1

Peculiar Velocity Model Parameters and Results

\begin{tabular}{|c|c|c|c|c|c|c|c|c|}
\hline \multirow[b]{2}{*}{ ModeL } & \multirow[b]{2}{*}{$\beta$} & \multirow{2}{*}{$\begin{array}{c}\boldsymbol{V} \\
\left(\mathrm{km} \mathrm{s}^{-1}\right)\end{array}$} & \multicolumn{3}{|c|}{$\Omega=1+$ BAO PRIOR } & \multicolumn{3}{|c|}{$w=-1+$ BAO PRIOR } \\
\hline & & & $w$ & $\Omega_{m}$ & $\chi_{w, \Omega_{m}}^{2}$ & $\Omega_{\Lambda}$ & $\Omega_{m}$ & $\chi_{\Omega_{\Lambda}, \Omega_{m}}^{2}$ \\
\hline $\mathrm{A} 06^{\mathrm{a}}$ & 0.0 & $\ldots$ & $-1.023 \pm 0.090$ & $0.271 \pm 0.021$ & $\cdots$ & $0.751 \pm 0.082$ & $0.271 \pm 0.020$ & \\
\hline NF $\ldots \ldots \ldots$ & 0.0 & $\ldots$ & $-1.054_{-0.084}^{+0.086}$ & $0.270_{-0.018}^{+0.024}$ & 115.5 & $0.770_{-0.071}^{+0.083}$ & $0.269_{-0.017}^{+0.033}$ & 115.4 \\
\hline PBF $\ldots . .$. & 0.0 & $57,-540,314$ & $-1.026_{-0.083}^{+0.085}$ & $0.273_{-0.019}^{+0.024}$ & 129.4 & $0.741_{-0.073}^{+0.084}$ & $0.273_{-0.017}^{+0.034}$ & 129.2 \\
\hline B05 ....... & $0.5^{\mathrm{b}}$ & $70,-194,0$ & $-1.081_{-0.085}^{+0.087}$ & $0.268_{-0.018}^{+0.024}$ & 110.3 & $0.796_{-0.070}^{+0.081}$ & $0.267_{-0.017}^{+0.032}$ & 110.1 \\
\hline B07 ........ & 0.7 & $\ldots$ & $-1.094_{-0.085}^{+0.087}$ & $0.267_{-0.018}^{+0.024}$ & 111.2 & $0.809_{-0.069}^{+0.082}$ & $0.265_{-0.017}^{+0.032}$ & 111.1 \\
\hline
\end{tabular}

${ }^{a}$ Results quoted in A06 marginalizing analytically over $\alpha_{s}$ and $\beta_{c}$ (see $\S 3$ ).

${ }^{\mathrm{b}}$ Best-fit value from Pike \& Hudson (2005).

the mass density. The peculiar velocity in the cosmic microwave background (CMB) frame is then given by linear perturbation theory (Peebles 1980) applied to the density field (see, e.g., Yahil et al. 1991; Hudson 1993):

$$
\boldsymbol{v}=\frac{\beta}{4 \pi} \int^{R_{\max }} \delta\left(\boldsymbol{r}^{\prime}\right) \frac{\left(\boldsymbol{r}^{\prime}-\boldsymbol{r}\right)}{\left|\boldsymbol{r}^{\prime}-\boldsymbol{r}\right|^{3}} d^{3} \boldsymbol{r}^{\prime}+\boldsymbol{V}
$$

In this Letter, we use the density field of the IRAS PSCz galaxies (Branchini et al. 1999), which extends to a depth $R_{\max }=20,000 \mathrm{~km} \mathrm{~s}^{-1}$. Contributions to the peculiar velocity arising from masses on scales larger than $R_{\max }$ are modeled by a simple residual dipole, $\boldsymbol{V}$. Thus, given a density field, the parameters $\beta$ and $\boldsymbol{V}$ describe the velocity field within $R_{\max }$. For galaxies with distances greater than $R_{\max }$, the first term above is set to zero.

The predicted peculiar velocities from the PSCz density field are subject to two sources of uncertainty: (1) the noisiness of the predictions due to the sparsely sampled density field and (2) the inapplicability of linear perturbation theory on small scales. Typically these uncertainties are accounted for by adding an additional "thermal" dispersion, which is assumed to be Gaussian. From a careful analysis of predicted and observed peculiar velocities, Willick \& Strauss (1998) estimated these uncertainties to be $\sim 100 \mathrm{~km} \mathrm{~s}^{-1}$, albeit with a dependence on density. Radburn-Smith et al. (2004) found reasonable $\chi^{2}$ values if $150 \mathrm{~km} \mathrm{~s}^{-1}$ was assumed in the field, with an extra contribution to the small-scale dispersion added in quadrature for $\mathrm{SNe}$ in clusters. Here we adopt a thermal dispersion of 150 $\mathrm{km} \mathrm{s}^{-1}$.

For this study, we explore the results of three different models of large-scale flows and compare them to a case where no flow model is used. These models have been chosen to span the range of flow models permitted by peculiar velocity data, and all of these models reproduce the observed $\sim 600 \mathrm{~km} \mathrm{~s}^{-1}$ motion of the Local Group with respect to the CMB. The first model assumes a pure bulk flow (model PBF; hence $\beta=0$ ), with $\boldsymbol{V}$ having vector components $(57,-540,314) \mathrm{km} \mathrm{s}^{-1}$ in Galactic Cartesian coordinates. The second model assumes $\beta=0.5$ (model B05), with a dipole vector of $(70,-194,0)$ $\mathrm{km} \mathrm{s}^{-1}$. The third model adopts $\beta=0.7$ (model B07), which requires no residual dipole. We compare these models to the no-correction scenario adopted by A06 and others with $\beta=$ 0 and $V=0$, which we call the "no-flow" or NF scenario. Note that a recent comparison (Pike \& Hudson 2005) of results from IRAS predictions versus peculiar velocity data yields a mean value fit with $\beta=0.50 \pm 0.02$ (stat), so the B05 model is strongly favored over the NF scenario by independent peculiar velocity analyses.

\section{COSMOLOGICAL FITS}

Prior to the fitting procedure, the peculiar velocities for each model are used to correct the local $\mathrm{SNe}$ (using a variation of eqs. [11] and [13] in Hui \& Greene 2006). We then fit our corrected SN data in two ways using a $\chi^{2}$-gridding cosmology fitter $^{1}$ (also used by Wood-Vasey et al. 2007). The first fit uses a flat cosmology $(\Omega=1)$ with the equation of state parameter $w$ and $\Omega_{m}$ as free parameters. The second fit assumes a $\Lambda \mathrm{CDM}$ ( $w=-1$ ) cosmology with $\Omega_{\Lambda}$ and $\Omega_{m}$ as free parameters. We used the same intrinsic SN photometric scatter $\left(\sigma_{\mathrm{int}}=0.13 \mathrm{mag}\right.$; A06) for every fit. The resulting $\chi^{2}$ probability surfaces for both fits are then further constrained using the baryon acoustic oscillation (BAO) result from Eisenstein et al. (2005). The final derived cosmological parameters are then used to calculate the $\chi^{2}$ for each fit (see A06, § 5.4).

The fitting procedure employed here differs in implementation from that used in A06. Three additional parameters, often called nuisance parameters, must be fit along with the two cosmological parameters. These parameters are the constant of proportionality for the SN light-curve shape, $\alpha_{s}$, the correction for the SN observed color, $\beta_{c}$, and a SN brightness normalization, $\mathcal{M}$. We distinguish $\beta_{c}$ from the $\beta$ used to describe the flow models above. A06 used analytic marginalization of the nuisance parameters $\alpha_{s}$ and $\beta_{c}$ in their fits. Here these parameters are fully gridded like the cosmological parameters. This avoids a bias in the nuisance parameters that results because, in the analytic method, their values must be held fixed to compute the errors. The result is that our fits using the NF scenario produce slightly different cosmological parameters than quoted in A06.

\section{RESULTS}

The results of the cosmological fits for each model are listed in Table 1 and plotted in Figures 1 and 2. They demonstrate two effects of the peculiar velocity corrections: a change in the values of the cosmological parameters and a change in the quality of the fits as measured by the $\chi^{2}$ statistic.

We expect, if a given model is correct, to improve the fitting since our corrected data should more closely resemble the homogeneous universe described by a few cosmological parameters. The $\chi^{2}$ of the fits for each flow model can be compared to the $\chi^{2}$ for the NF scenario (shown by the dashed lines in the figures) as a test of this hypothesis. Using $\Delta \chi^{2}=-2 \ln \left(L / L_{\mathrm{NF}}\right)$, where $L$ is the likelihood, we find that the pure bulk flow is over $10^{3}$ times less likely than the NF scenario, while the B05 and B07 models are 13.5 and 8.6 times more likely, respectively.

\footnotetext{
${ }^{1}$ See http://qold.astro.utoronto.ca/conley/simple_cosfitter/.
} 


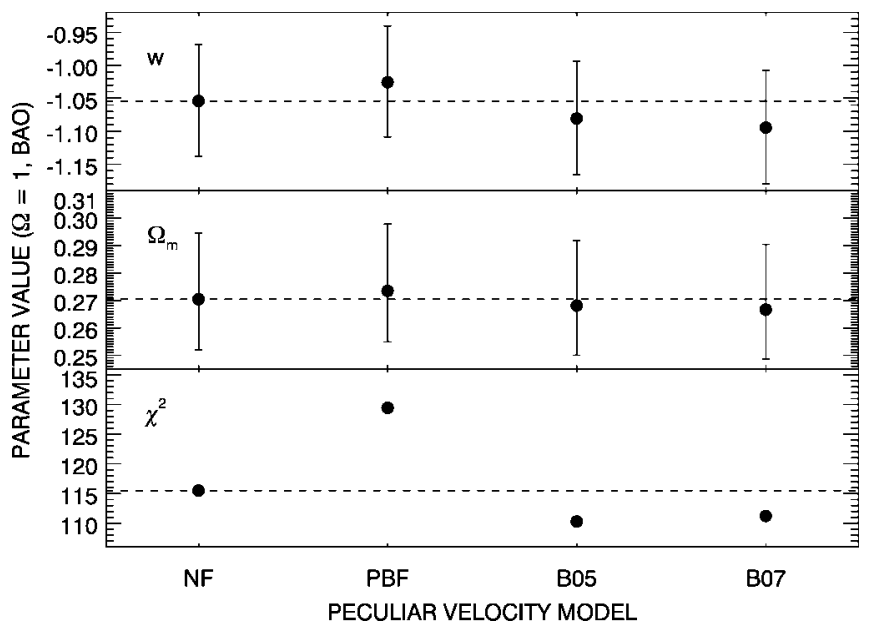

FIG. 1.-Parameter values for the $w, \Omega$ fit $(\Omega=1+$ BAO prior) for each of the four peculiar velocity models in Table 1 . The values for the NF scenario are indicted by the dashed lines. The largest systematic error in $w$ compared with the NF fit is +0.040 for the B07 model, which demonstrates the amplitude of the systematic error if peculiar velocity is not accounted for. The offsets for $\Omega_{m}$ are all within \pm 0.003 , showing that this parameter is not sensitive to the peculiar velocity corrections due to the BAO prior. The $\chi^{2}$ of the fits improve when using the two $\beta$ models (B05, B07), while the PBF model provides a significantly worse fit.

We also use these data to assess the systematic errors made in the parameters if no peculiar velocities are accounted for. The largest of these are obtained by comparing the B07 model with the NF scenario. This comparison yields $\Delta w_{\mathrm{B} 07}=+0.040$ and $\Delta \Omega_{\Lambda, \text { B07 }}=-0.039$. The same comparison for the B05 model, which is only slightly preferred by the $\chi^{2}$ statistic over model B07, produces $\Delta w_{\mathrm{B} 05}=+0.027$ and $\Delta \Omega_{\Lambda, \text { в05 }}=-0.026$. The systematic offsets for $\Omega_{m}$ are all 0.004 or less, demonstrating the insensitivity of this parameter to peculiar velocities. This is due to the BAO prior, which is insensitive to local flow and provides a much stronger constraint for $\Omega_{m}$ than for either $w$ or $\Omega_{\Lambda}$ (see A06, Figs. 5 and 6).

\section{DISCUSSION AND SUMMARY}

The systematic effect of different flow models is at the level of \pm 0.04 in $w$. This is smaller than the present level of random error in $w$, which is largely due to the small numbers of highand low-redshift SNe. However, compared to other systematics discussed in A06, which total $\Delta w= \pm 0.054$, the systematic effect of large-scale flows is important. In their Table 5, WoodVasey et al. (2007) list 16 sources of systematic error that total $\Delta w= \pm 0.13$. Aside from three method-dependent systematics and the photometric zero-point error, they are all smaller than the flow systematic. As the number of SNe continues to increase, and understanding of other systematics (e.g., photometric zero points) improves, it is possible that large-scale flows will become one of the dominant sources of systematic uncertainty.

The peculiar velocities of SN host galaxies arise from largescale structures over a range of scales. The component arising from small-scale, local structure is the least important: it is essentially a random variable that is reduced by $\sqrt{N}$. More problematic is the large-scale coherent component. Such a largescale component can take several forms: an overdensity or underdensity; a large-scale dipole; or "bulk" flow.

The existence of a large-scale but local $\left(<7400 \mathrm{~km} \mathrm{~s}^{-1}\right)$ underdensity, or "Hubble bubble," was first discussed by Zehavi

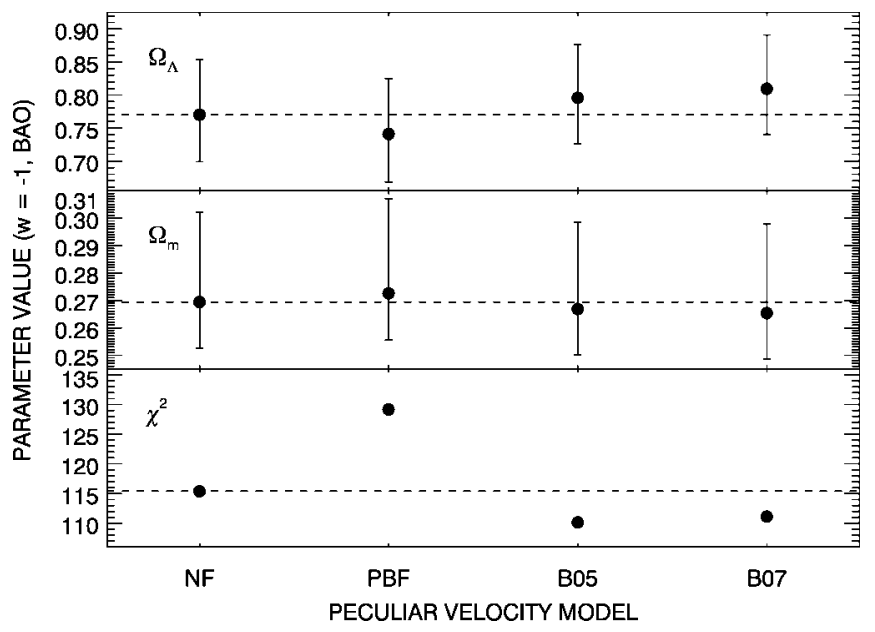

FIG. 2.-Parameter values for the $\Omega_{\Lambda}, \Omega_{m}$ fit ( $w=-1+$ BAO prior) for each of the four peculiar velocity models as in Fig. 1. Again, comparing the $\mathrm{NF}$ fits to the B07 model produces the largest systematic in $\Omega_{\Lambda}$ of -0.039 . We also find $\Omega_{m}$ insensitive to the corrections, having all offsets within \pm 0.004 . The $\chi^{2}$ values show the same pattern as in Fig. 1, favoring the $\beta$ models over no correction (NF) and over pure bulk flow.

et al. (1998). Recently, Jha et al. (2007) have reenforced this claim with a larger SN data set; they find that the difference in the Hubble constant inside the bubble and outside is $\Delta H / H=6.5 \% \pm 1.8 \%$. If correct, this could have a dramatic effect on the derived cosmological parameters (Jha et al. 2007, Fig. 17), especially for those studies that extend their local sample down below $z<0.015$. However, the "Hubble bubble" was not confirmed by Giovanelli et al. (1999), who found $\Delta H / H=1.0 \% \pm 2.2 \%$ using the Tully-Fisher (TF) peculiar velocities, or by Hudson et al. (2004), who found $\Delta H / H=$ $2.3 \% \pm 1.9 \%$ using the fundamental plane (FP) distances.

According to equation (1), a mean underdensity of IRAS galaxies of order $\sim 40 \%$ within $7400 \mathrm{~km} \mathrm{~s}^{-1}$ would be needed to generate the "Hubble bubble" quoted by Jha et al. (2007). However, we find that the IRAS PSCz density field of Branchini et al. (1999) is not underdense in this distance range; instead, it is mildly overdense (by a few percent) within $7400 \mathrm{~km} \mathrm{~s}^{-1}$ (see also Branchini et al. 1999, Fig. 2). As a further crosscheck, when we refit the Jha et al. (2007) data after having subtracted the predictions of the B05 flow model, the "bubble" remains in the Jha et al. (2007) data. Thus, the Jha et al. "bubble" cannot be explained by local structure, unless that structure is not traced by IRAS galaxies. Moreover, when we analyze the $99 \mathrm{SNe}$ within $15,000 \mathrm{~km} \mathrm{~s}^{-1}$ from Tonry et al. (2003) in the same way, we find no evidence of a significant "Hubble bubble" $(\Delta H / H=1.5 \% \pm 2.0 \%)$, in agreement with the results from TF and FP surveys. The Tonry et al. (2003) sample and that of Jha et al. (2007) have $67 \mathrm{SNe}$ in common. The high degree of overlap suggests that the difference lies in the different methods for converting the photometry into SN distance moduli.

A local large-scale flow can also introduce systematic errors if the low- $z$ sample is biased in its sky coverage; in this case, an uncorrected dipole term can corrupt the monopole term, which then biases the cosmological parameters. For the largescale flow directions considered here, this does not appear to affect the A06 sample; we note that the PBF-corrected case has similar cosmological parameters to the "no-flow" case. However, if coherent flows exist on large scales, this may affect 
surveys with unbalanced sky coverage, such as the SN Factory (Aldering et al. 2002) or the SDSS SN survey. ${ }^{2}$

The most promising approach to treating the effect of largescale flows is a more sophisticated version of the analysis presented here: combine low-redshift $\mathrm{SNe}$ with other low-redshift

${ }^{2}$ See http://sdssdp47.fnal.gov/sdsssn/sdsssn.html. peculiar velocity tracers, such as Tully-Fisher SFI ++ survey (Masters et al. 2006) and the NOAO Fundamental Plane Survey (Smith et al. 2004), and use these data to constrain the parameters of the flow model ( $\beta$ and the residual large-scale flow $\boldsymbol{V})$ directly. One can then marginalize over the parameters of the flow model while fitting the cosmological parameters to the low- and high- $z$ SNe.

\section{REFERENCES}

Albrecht, A., et al. 2006, preprint (astro-ph/0609591)

Aldering, G., et al. 2002, in Proc. SPIE 4836, 61

Altavilla, G., et al. 2004, MNRAS, 349, 1344

Astier, P., et al. 2006, A\&A, 447, 31 (A06)

Branchini, E., et al. 1999, MNRAS, 308, 1

Cooray, A., \& Caldwell, R. R. 2006, Phys. Rev. D, 73, 103002

Eisenstein, D. J., et al. 2005, ApJ, 633, 560

Giovanelli, R., Dale, D. A., Haynes, M. P., Hardy, E., \& Campusano, L. E. 1999, ApJ, 525, 25

Hamuy, M., Phillips, M. M., Suntzeff, N. B., Schommer, R. A., Maza, J., \& Aviles, R. 1996, AJ, 112, 2391

Haugboelle, T., Hannestad, S., Thomsen, B., Fynbo, J., Sollerman, J., \& Jha, S. 2006, ApJ, submitted (astro-ph/0612137)

Hudson, M. J. 1993, MNRAS, 265, 43

. 2003, preprint (astro-ph/0311072)

Hudson, M. J., Smith, R. J., Lucey, J. R., \& Branchini, E. 2004, MNRAS, 352,61

Hui, L. \& Greene, P. B. 2006, Phys. Rev. D, 73, 123526

Jha, S. 2002, Ph.D. thesis, Harvard Univ.

Jha, S., Riess, A. G., \& Kirshner, R. P. 2007, ApJ, 659, 122

Krisciunas, K., et al. 2001, AJ, 122, 1616

. 2004a, AJ, 127, 1664
Krisciunas, K., et al. 2004b, AJ, 128, 3034

Masters, K. L., Springob, C. M., Haynes, M. P., \& Giovanelli, R. 2006, ApJ, 653, 861

Peebles, P. J. E. 1980, The Large-Scale Structure of the Universe (Princeton: Princeton Univ. Press)

Perlmutter, S., et al. 1999, ApJ, 517, 565

Pike, R. W., \& Hudson, M. J. 2005, ApJ, 635, 11

Radburn-Smith, D. J., Lucey, J. R., \& Hudson, M. J. 2004, MNRAS, 355, 1378

Riess, A. G., et al. 1998, AJ, 116, 1009 1999, AJ, 117, 707 2007, ApJ, 659, 98

Sarkar, D., Feldman, H. A., \& Watkins, R. 2007, MNRAS, 375, 691

Smith, R. J., et al. 2004, AJ, 128, 1558

Strolger, L.-G., et al. 2002, AJ, 124, 2905

Tonry, J. L., et al. 2003, ApJ, 594, 1

Watkins, R., \& Feldman, H. A. 2007, preprint (astro-ph/0702751)

Willick, J. A., \& Strauss, M. A. 1998, ApJ, 507, 64

Wood-Vasey, W. M., et al. 2007, ApJ, submitted (astro-ph/0701041)

Yahil, A., Strauss, M. A., Davis, M., \& Huchra, J. P. 1991, ApJ, 372, 380

Zehavi, I., Riess, A. G., Kirshner, R. P., \& Dekel, A. 1998, ApJ, 503, 483 